\title{
Lymphatic deletion of calcitonin receptor-like receptor exacerbates intestinal inflammation
}

\author{
Reema B. Davis, Daniel O. Kechele, Elizabeth S. Blakeney, John B. Pawlak, and Kathleen M. Caron \\ Department of Cell Biology and Physiology, University of North Carolina, Chapel Hill, North Carolina, USA.
}

\begin{abstract}
Lymphatics play a critical role in maintaining gastrointestinal homeostasis and in the absorption of dietary lipids, yet their roles in intestinal inflammation remain elusive. Given the increasing prevalence of inflammatory bowel disease, we investigated whether lymphatic vessels contribute to, or may be causative of, disease progression. We generated a mouse model with temporal and spatial deletion of the key lymphangiogenic receptor for the adrenomedullin peptide, calcitonin receptor-like receptor (Calcrl), and found that the loss of lymphatic Calcrl was sufficient to induce intestinal lymphangiectasia, characterized by dilated lacteals and protein-losing enteropathy. Upon indomethacin challenge, Calcr $\left.\right|^{f / f / f} /{\text { Prox } 1-C r e E R^{T 2}}$ mice demonstrated persistent inflammation and

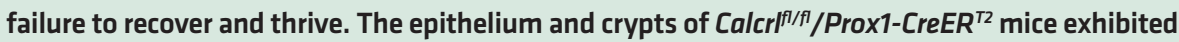
exacerbated hallmarks of disease progression, and the lacteals demonstrated an inability to absorb lipids. Furthermore, we identified Calcrl/adrenomedullin signaling as an essential upstream regulator of the Notch pathway, previously shown to be critical for intestinal lacteal maintenance and junctional integrity. In conclusion, lymphatic insufficiency and lymphangiectasia caused by loss of lymphatic Calcrl exacerbates intestinal recovery following mucosal injury and underscores the importance of lymphatic function in promoting recovery from intestinal inflammation.
\end{abstract}

Conflict of interest: The authors have declared that no conflict of interest exists.

Submitted: December 22, 2016 Accepted: February 14, 2017 Published: March 23, 2017

Reference information: JCI Insight. 2017;2(6):e92465. https:// doi.org/10.1172/jici.insight.92465.

\section{Introduction}

Lymphatic vessels are highly prevalent in most tissues throughout the body and play a major role in regulating interstitial fluid balance, immune cell trafficking, and dietary fat absorption (1). Within the intestinal tract, each intestinal villus contains a lymphatic lacteal that efficiently absorbs lipids that have been packaged as chylomicrons by enterocytes (2-4). These intestinal lacteals then drain the lipid-rich chyle fluid to submucosal collecting lymphatic vessels for systemic fat absorption. The critical importance of lymphatic vessels in intestinal biology was recently demonstrated by diphtheria toxin-mediated ablation of lymphatic vessel endothelial hyaluronan receptor 1-expressing (LYVE1-expressing) lymphatic endothelial cells (LECs) in the adult mouse intestine, leading to sepsis-related lethality due to failed immune cell trafficking and breakdown of the intestinal barrier to gut microbiota (5). In addition, a few recent studies have shown that lymphatic growth factors, such as intestinal VEGF-D and smooth muscle-derived VEGF-C, govern lacteal maintenance and dietary lipid absorption and that lacteals are continuously regenerating via VEGFR2- and VEGFR3-induced delta-like 4 (DLL4) signaling $(6,7)$

Despite the importance of the intestinal lymphatic system in immune surveillance and maintaining the intestinal barrier against microbes, the pathological versus protective roles of lymphatics in inflammatory bowel diseases (IBDs) still remain vastly elusive (8). IBD encompasses both ulcerative colitis, which affects the colon, and Crohn's disease (CD), which primarily affects the distal ileum. Obstructed intestinal lymphatics are an established histological hallmark of $\mathrm{CD}$, which stands somewhat in contrast to the lymphatic expansion observed in the mucosa of patients with IBDs (9-13), because it remains unclear whether lymphatic insufficiency due to obstruction is the cause of IBD or whether excessive lymphatic growth results from lymphatic insufficiency. In postoperative CD patients, a low density of lymphatic vessels has been attributed to recurrence of the disease (14), and an inability of lymphatic vessels to function has been associated with lymphedema of the intestinal walls in IBD patients (15). In animal models, VEGF-C administration was shown to stimulate lymphatic function and abrogate experimental colitis $(16,17)$. Counterintuitively, it was also demonstrated by this same group that increases in VEGF-C-induced lymphangiogenesis 
did not promote disease resolution in mice with acute experimental colitis (18). Collectively, these results suggest either that lymphatic insufficiency may be causative of IBDs or that incorrect lymphatic expansion and function can exacerbate the disease phenotype. Although there are some large animal models, including several inbred strains of dogs, that exhibit intestinal lymphatic insufficiency, development of genetically tractable animal model systems in which the causes and consequences of lymphatic insufficiency can be addressed in the context of inflammation initiation and progression is needed $(3,19)$.

Adrenomedullin (AM) is a small multifunctional peptide that signals through a heterodimer of a GPCR, calcitonin receptor-like receptor (gene $=C A L C R L$, protein $=\mathrm{CLR}$ ), and receptor activity-modifying protein 2 (RAMP2). These signaling partners are crucial for embryonic development since genetic deletion of Calcrl, $\mathrm{Adm}$, or Ramp2 causes midgestation lethality due to arrested lymphangiogenesis in mice (20-22). In adult mice, the inducible, global genetic deletion of the Calcrl gene causes systemic lymphatic insufficiency in a multitude of lymphatic vascular beds, leading to corneal edema, distal limb edema, and lymphangiectasia (23). Since CLR is expressed in many cell types and tissues, we developed a genetic mouse model with inducible deletion of the Calcrl gene in lymphatic endothelium. Consistently, we found that loss of lymphatic Calcrl is sufficient to induce systemic lymphatic insufficiency and intestinal lymphangiectasia. Therefore, in this present study, challenging these mice with acute inflammation, we demonstrate that lymphatic CLR is not only important in maintaining functional intestinal lymphatic vessels but also critical in the pathophysiology of small intestinal inflammation, disease progression, and recovery.

\section{Results}

Lymphatic deletion of Calcrl is sufficient to induce small intestinal lymphangiectasia. Global deletion of the gene coding for the AM receptor, Calcrl, has been shown to induce multiorgan lymphangiectasia, resulting from dilated lymphatic vessels across several vascular beds (23). Since Calcrl is commonly expressed in several cell types, including cells outside the vasculature, to distinguish the role of Calcrl in lymphatic cells compared with its role in other cell types in contributing to lymphangiectasia, we deleted Calcrl from the adult mouse lymphatic endothelium. We crossed Calcrll/fl mice to the previously described Prox1-CreER ${ }^{T 2}$ line (24). Five consecutive tamoxifen (TAM) injections (Supplemental Figure 1A; supplemental material available online with this article; https://doi.org/10.1172/jci.insight.92465DS1) in sex- and age-matched mice resulted in Calcrl deletion, as detected by an excision band by PCR (Supplemental Figure 1B), and a significant $70 \%$ reduction in Calcrl gene expression, as detected by quantitative RT-PCR of lungs from Calcrl/ff/Prox1-CreER $R^{T 2}$ mice compared with TAM-injected Calcrt ${ }^{\text {flfl }}$ controls (Figure 1A). In the normal adult mouse intestinal tract, PROX1 has been shown to be expressed either in a subset of mature enteroendocrine cells or $\mathrm{Ngn}^{+}$enteroendocrine cells going through terminal differentiation or in their long-lived enteroendocrine progenitors $(25,26)$. Therefore, to confirm the cell-specific expression of Prox1-CreER ${ }^{T 2}$, RiboTag technology was applied (27). An intestinal enterocyte fraction was separated from the nonenterocyte fraction using TAM-injected WT/Proxl-CreER ${ }^{T 2} /$ $R p l 22^{\text {tm1.1Psam }}$ (with WT Calcrl) and Calcrt ${ }^{\text {tlfl }} /$ Prox1-CreER $R^{T 2} / R p l 22^{\text {tm1.1Psam }}$ mice, and Cre-expressing cell-specific ribosome-associated RNA was harvested and validated for Calcrl downregulation. Calcrl expression was significantly downregulated over 90-fold in the nonenterocyte fraction of TAM-injected Calcrl/f/fl/Prox1-CreER ${ }^{T 2} /$ $R p l 22^{\text {tml.1Psam }}$ mice and remained unchanged in the enterocyte fraction compared with TAM-injected WT/ Prox1-CreER $R^{T 2} / R p l 22^{t m 1.1 P s a m}$ mice (Figure 1B). In addition, staining of WT/Prox1-CreER $R^{T 2} / R p l 22^{\text {tml.1Psam }}$ ileum using an HA antibody shows that Prox1-CreER ${ }^{T 2}$ was expressed primarily in the intestinal lymphatic vessels (Supplemental Figure 1C, arrows) and few enteroendocrine cells (Supplemental Figure 1C, arrowheads). Collectively, in the absence of a reliable CLR antibody, these data demonstrate a robust and specific loss of Calcrl in intestinal lymphatics using the RiboTag technology (27).

Significant changes in body weight were observed in Calcrt ${ }^{7 l f l} /$ Proxl-CreER ${ }^{T 2}$ mice within 48 hours after the TAM injections as compared with control animals (Figure 1C). Serum albumin levels were significantly downregulated in Calcrl $^{7 / f t} /$ Proxl-CreER ${ }^{T 2}$ animals (Figure 1D), characteristic of protein-losing enteropathy. The Cal$\mathrm{crl}^{l / f l} /$ Prox1-CreER ${ }^{T 2}$ mice exhibited dilated lacteals and submucosal lymphatic capillaries in their small intestines upon TAM injection (Figure 2, A-H, arrows). Lacteal cross-sectional area was significantly larger in $\mathrm{Calcrl}^{\text {t/fl } /}$ Prox1-CreER ${ }^{T 2}$ animals compared with uninjected and TAM-injected controls (Figure 2, I-M, arrows). These data demonstrate that lymphatic deletion of CLR is sufficient to induce intestinal lymphangiectasia.

To address whether mice were acutely ill after prolonged loss of Calcrl, we studied TAM-injected Calcr $t^{7 \prime}$ fl/CAGGCre-ER ${ }^{T M}$ mice (with global inducible loss of Calcrl). We found that these mice were able to survive over 1.5 years, and their small intestines did not demonstrate any basal inflammation (Supplemental Figure 


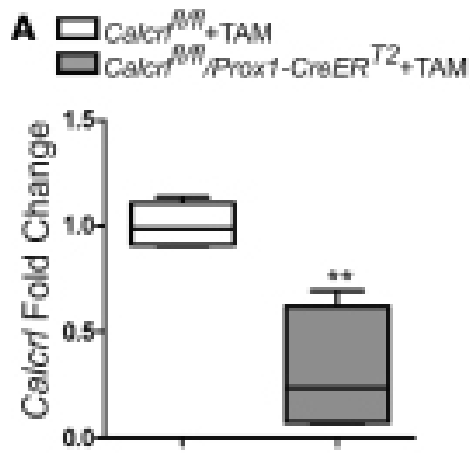

B
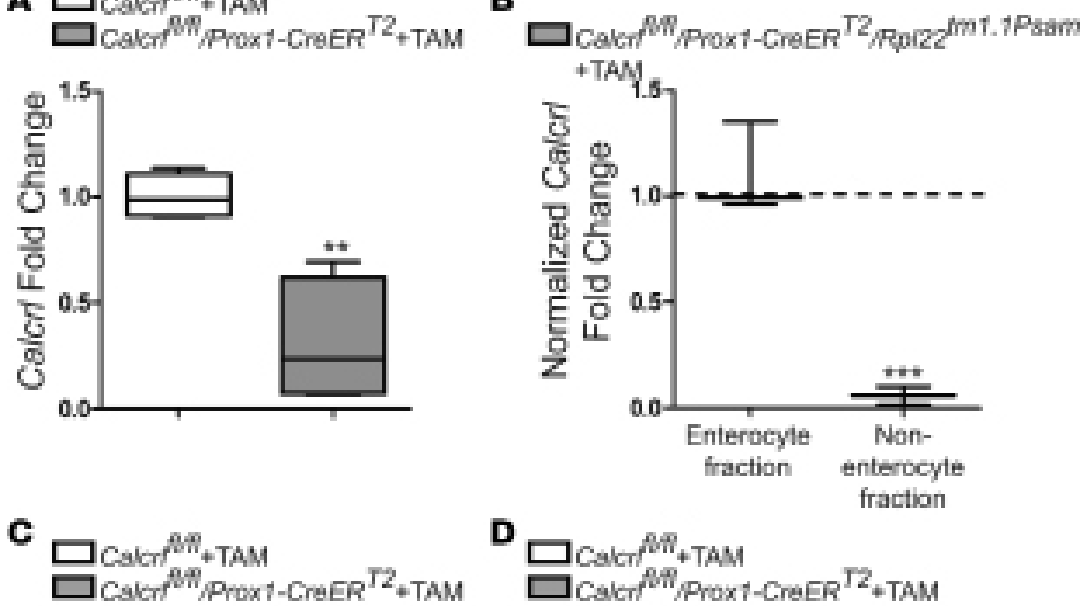

D

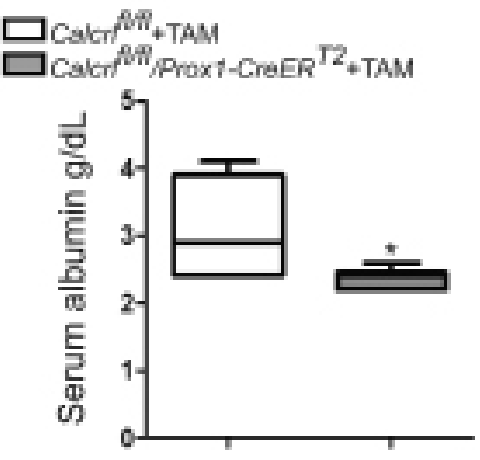

Figure 1. Loss of Calcrl using Prox1-CreER ${ }^{\text {T2 }}$ leads to reduced weight gain and protein loss. (A) Relative expression of Calcrl in lungs of tamoxifen-treated (TAM-treated)

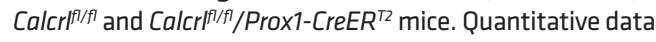
are represented as a box-and-whisker plot, with bounds from 25th to 75 th percentile, median line, and whiskers ranging from minimum to maximum values of fold change over Calcr $\left.\right|^{f / 7 f}$ controls; $n=7-8$ animals in each group. Gapdh was used as housekeeping control. Significance was determined by 2 -tailed, type 2 Student's $t$ test, ${ }^{* *} P<0.01$. (B) Relative expression of Calcrl in intestinal enterocyte and nonenterocyte population of TAM-treated WT/ Prox1-CreER ${ }^{\text {T2 }} /$ Rpl22 $^{\text {tm1.1.Psam }}$ and Calcr fl/f/Prox1-CreER $^{\text {T2 } / ~}$ $R p / 22^{\text {tmm.1.1Psam }}$ mice. Quantitative data are represented as a box-and-whisker plot, with bounds from 25 th to 75 th percentile, median line, and whiskers ranging from minimum to maximum values of normalized fold change over WT/ Prox1-CreER ${ }^{\text {T2 }} /$ Rpl22 ${ }^{\text {tm1.1Psam }}$ controls (dashed line); $n=3$ animals in each group. Gapdh was used as housekeeping control. Significance was determined by 2-way ANOVA, ${ }^{* * *} P<0.001$. (C and D) Measurement of weight loss (C)

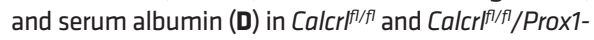
CreER ${ }^{T 2}$ mice after TAM treatment. Quantitative data are represented as a box-and-whisker plot, with bounds from 25 th to 75 th percentile, median line, and whiskers ranging from minimum to maximum values for percentage weight loss (C) and serum albumin (D). $n=5$ for Calcr $\left.\right|^{f / / 7}$ and 4 for Calcr $^{f^{1 / f} / \text { Prox1-CreER }}{ }^{T 2}$ group. Significance was determined by 2 -tailed, type 2 Student's t test, ${ }^{*} P<0.05$.

2A). To determine whether mice in our study suffered from additional systemic insufficiencies, we looked at

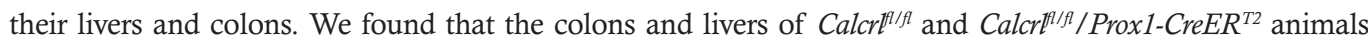
after TAM injection appeared structurally normal by H\&E (Supplemental Figure 2, B-F). Taken together, these results indicate that, under the conditions of Calcrl-mediated lymphatic insufficiency, mice exhibited prolonged survival and overall systemic health in the absence of any injury or challenge. In addition, the lack of any obvious liver pathology supports the interpretation that the protein-losing enteropathy phenotype (Figure 1D) is primarily a result of intestinal insufficiency. Therefore, these TAM-injected Calcrt/ff/Proxl-CreER $R^{T 2}$ mice now provide us with a genetic model of lymphatic insufficiency that can be explored to study the pathophysiology of disease progression and resolution after injury.

Calcrtllfl $_{\text {Prox1-CreER }}^{T 2}$ animals exhibit exacerbated intestinal damage and persistent inflammation following acute mucosal injury challenge. AM signaling in LECs plays an antiinflammatory role by suppressing inflammatory markers, such as TNF- $\alpha$, IL-8, and chemokine ligands $1,2,3$, and 20, in vitro (28). In addition, we have previously shown that AM signaling through the CLR/RAMP2 receptor complex can dampen systemic inflammatory responses (21). Since loss of lymphatic CLR gives rise to intestinal lymphangiectasia (Figures 1 and 2), we wanted to use this model to determine the extent to which lymphatic vessels contribute to pathophysiological disease progression and recovery after injury and evaluate the antiinflam-

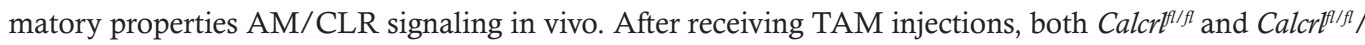
Prox1-CreER ${ }^{T 2}$ mice were challenged with indomethacin (INDO) - an established method to induce severe enteropathy in animals that recapitulates human CD by increasing mucosal permeability causing inflammation of the small intestine $(29,30)$. Mice were evaluated either 1 day after INDO, to study the acute degree of injury, or after 7 days to assess disease resolution (Supplemental Figure 3A).

Compared with Calcrlflfl controls, the small intestines of the Calcr $f^{f l / f l} /{\text { Prox } 1-C r e E R^{T 2}}$ animals were congealed and difficult to dissect. Macroscopic evaluation revealed visible, hemorrhagic lesions that persisted 7 days after INDO (Figure 3A, bottom inset). One day after INDO in both the control and Calcrflffl $^{\text {Prox 1-CreER }}{ }^{T 2}$ animals, histological examination of the intestines showed classical hallmarks of acute intestinal inflammation (31), including villous blunting, elongation of crypts, infiltration of inflammatory cells in the mucosa and submucosa, and thickening of the submucosa (Figure 3B). Importantly, the histological signs of inflammation markedly resolved in $\mathrm{Calcr}^{\mathrm{fl} / \mathrm{fl}}$ animals but persisted 


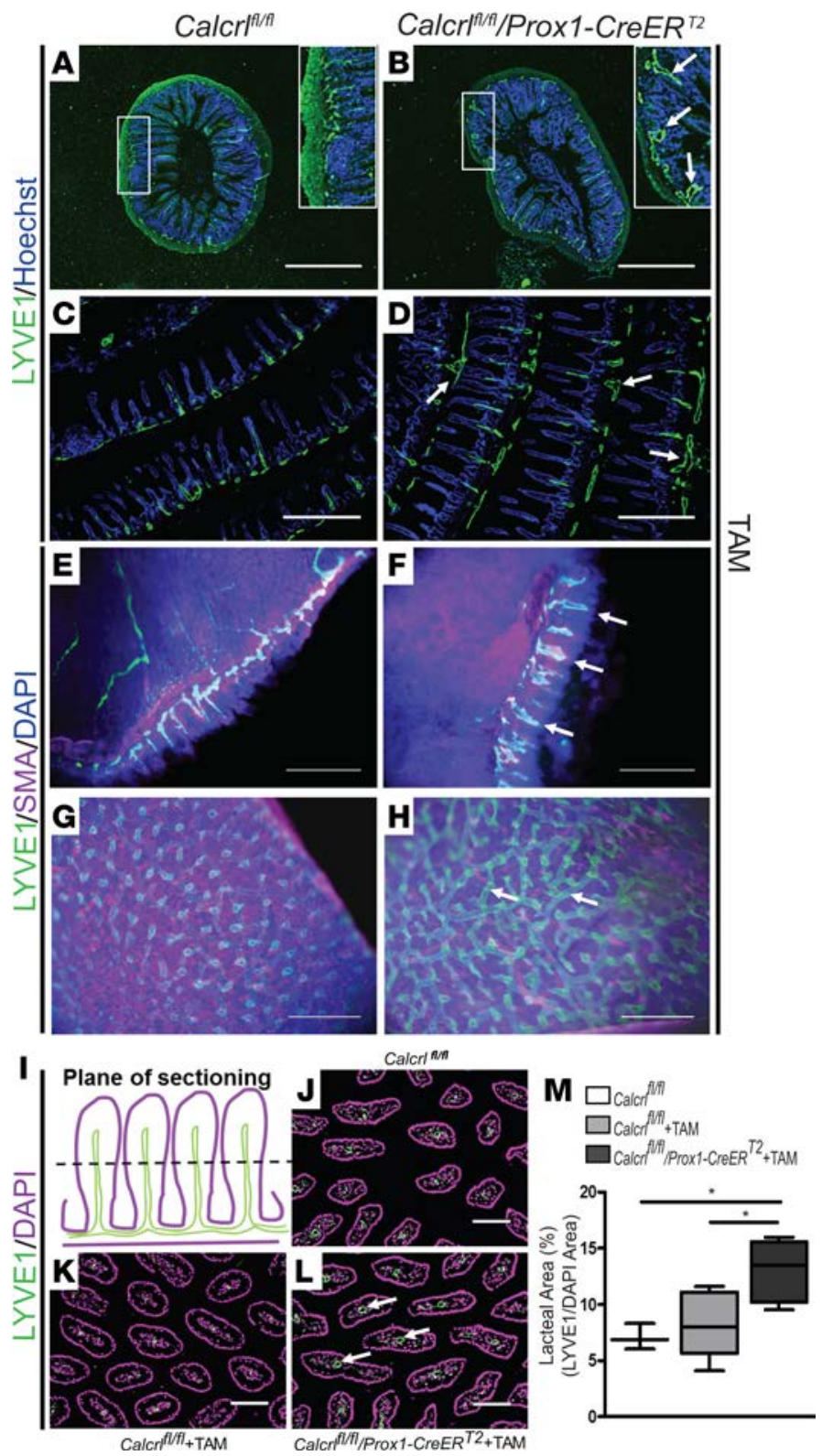

Figure 2. Lymphatic loss of Calcrl is sufficient to cause dilated lacteals and submucosal lymphatics. (A-D) Representative images of lymphatic vessels stained with lymphatic vessel endothelial hyaluronan receptor 1 (LYVE1) in

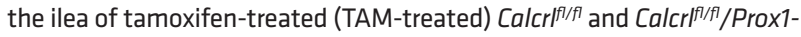
CreER $R^{T 2}$ mice. $n=3$ each group. Scale bar: $500 \mu \mathrm{m}$. Arrows point to dilated lymphatic vessels. (E-H) Representative whole mount LYVE1 and smooth muscle actin-stained (SMA-stained) ilea from TAM-treated Calcr $f^{1 / f f}$ and Calcr $^{f / f / f} /$ Prox1-CreER $^{T 2}$ animals. Scale bar: $500 \mu \mathrm{m}$. Arrows point to dilated lymphatic vessels. (I) Simplified schematic indicating the plane of sectioning (dashed line) of villi for J-L. (J-L) LYVE1 staining of jejunal lymphatic vessels

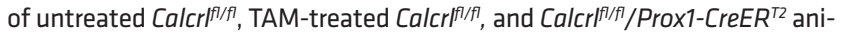
mals. Arrows point to dilated lymphatic vessels toward the center of the villi. Scale bar: 1,000 $\mu \mathrm{m}$. (M) The area of open lacteals normalized to the area of villi cross sections and expressed as a percentage. $n=3-5$ mice per group, with 20-60 villi quantified per mouse. Quantitative data are represented as a box-and-whisker plot, with bounds from 25th to 75th percentile, median line, and whiskers ranging from minimum to maximum values for percentage lacteal area. Significance was determined by 1-way ANOVA with Tukey's Multiple comparison test, ${ }^{*} P<0.05$.

in the Calcrtl/fl/Prox1-CreER ${ }^{T 2}$ mice following the 7-day recovery period (Figure 3B). Calcrtifl/Prox $1-C r e E R^{T 2}$ mice also had increased fibrosis in their small intestinal tissue (Figure 3C). Expression analysis of inflammatory markers, such as TNF- $\alpha$, $I L-6$, and $M P O$, showed significantly increased levels in Calcr $f^{f l}$ fl/Prox 1-CreER ${ }^{T 2}$ mice 7 days after INDO compared with controls, further indicative of persistent inflammation and impaired recovery (Figure 3D). The overall length of small intestines, as measured from proximal duodenum to distal ileum, was significantly reduced in TAM-injected Calcrf ${ }^{f l f l} / \operatorname{Prox} 1-\mathrm{CreER}^{T 2}$ mice 7 days after INDO challenge (Figure 3E). Calcrfl/ft/Prox1-CreER ${ }^{T 2}$ mice displayed significantly higher histopathological scores of inflammation, increased numbers of goblet cells per villus, increased crypt depth, and fibrosis 7 days after INDO challenge, unlike Calcrfl/fl mice, which recovered (Figure 3F; Supplemental Figure 3, B-H; and Table 1). These results indicate that lymphatic insufficiency in Calcr $f^{f l f l} /$ Prox1-CreER ${ }^{T 2}$ animals impairs intestinal recovery following drug-induced acute mucosal injury, highlighting the importance of functional lymphatics and CLR signaling in inflammation resolution.

Intestinal inflammation in mice lacking lymphatic Calcrl induces proliferation of crypts but not lacteal endothelial cells. The persistence of inflammation, villus blunting, and elongation of crypts upon INDO challenge in mice lacking lymphatic CLR signaling prompted us to examine the status of cellular proliferation and apoptosis of intestinal tissue. TAM-injected Calcrflffl/Prox1-CreER ${ }^{T 2}$ mice exhibited increased proliferation in the intestinal crypt epithelium after INDO challenge, as evidenced by increased phospho-histone H3 staining (Figure 4A). Subsequently, the villus epithelium had substantially more cleaved caspase-3 apoptotic cells in Calcrfl/fl/Prox1-CreER ${ }^{T 2}$ mice compared with controls (Figure 4B). As expected with the genetic loss of a critical lymphangiogenic signaling cue, we observed markedly reduced proliferation of the lymphatic endothelial lacteals in Calcrllffl/Prox1-CreER ${ }^{T 2}$ mice compared with controls (Figure 4C and Supplemental Figure 4, A and B). Nevertheless, increased proliferation in the crypts, in an attempt to regenerate villi, did not appear to aid in tissue recovery in the Calcrl/ffl/Prox $1-C_{\text {reER }}{ }^{T 2}$ mice 7 days after INDO. These data imply that, even under conditions of enhanced intestinal epithelial regeneration, the maintenance of lacteal growth and proliferation through CLR signaling is an essential component of appropriate tissue recovery following intestinal inflammation.

Loss of lymphatic CLR reorganizes adherens junctions and restricts chylomicron uptake. Intestinal lymphatic vessels are important for dietary fat absorption, and intestinal lymphangiectasia alters lipid 
A
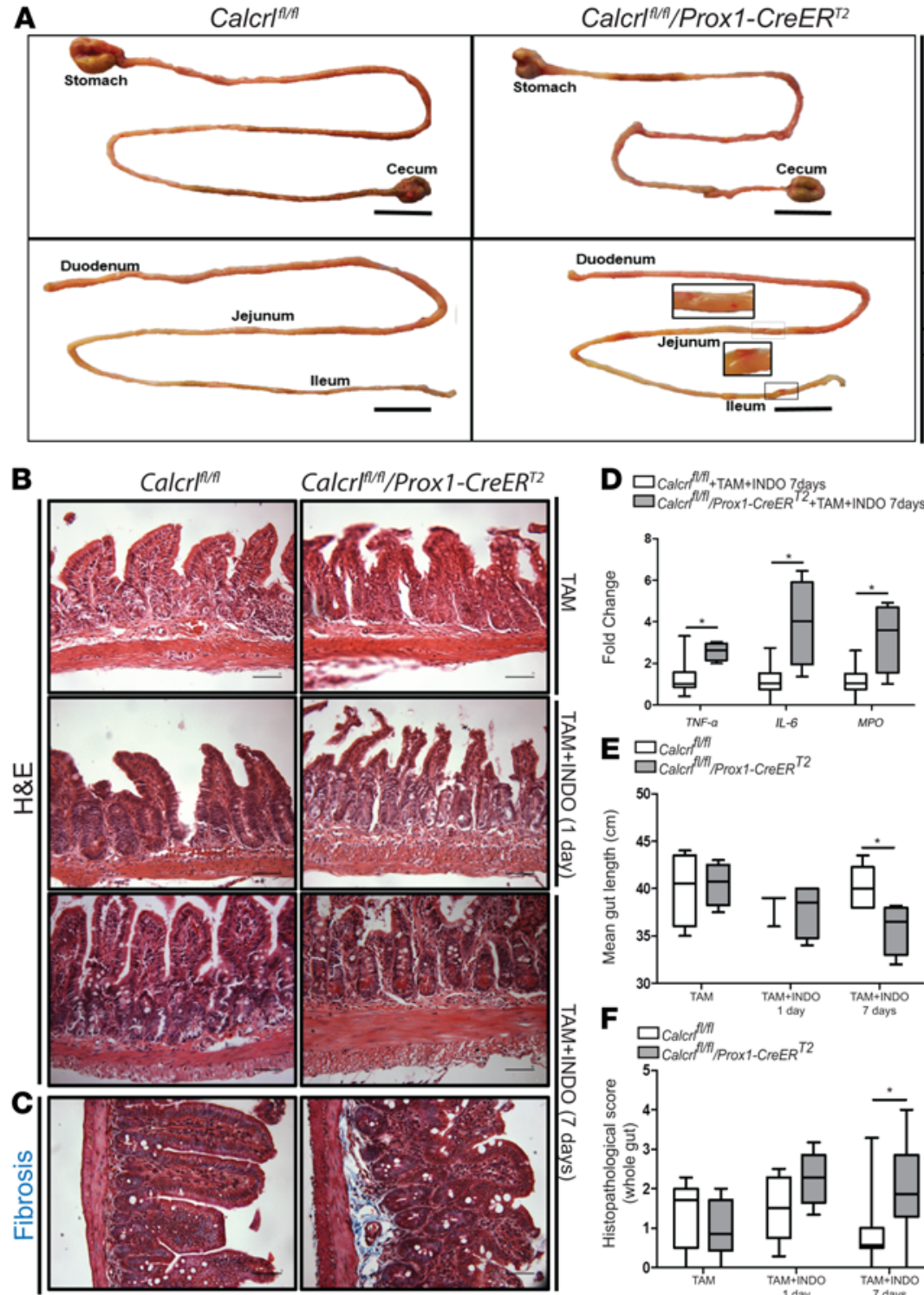

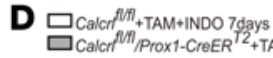
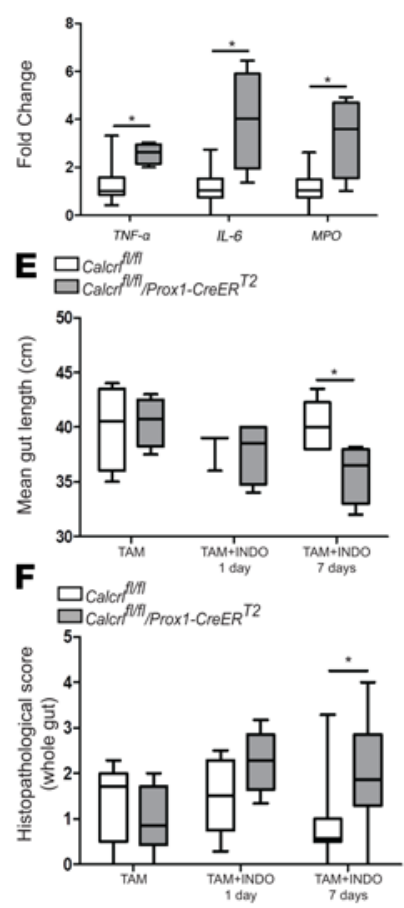

Figure 3. Mice lacking lymphatic Calcrl do not recover from an acute mucosal injury challenge. (A) Representative images from whole small intestine (stomach and cecum attached) from tamoxifen-treated (TAM-treated)

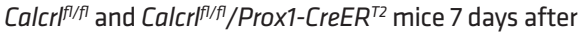
indomethacin (INDO) challenge. $n=7-8$ animals in each group. Bottom panels show flushed intestines (stomach and cecum removed), and boxed areas are expanded as insets to show spot lesions. Scale bar: 1 $\mathrm{cm}$. (B) Representative images of $\mathrm{H} \& \mathrm{E}$-stained ilea

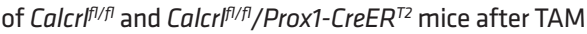
treatment, 1 day after INDO challenge, and 7 days after INDO challenge. $n=5-10$ animals in each group for each treatment. Scale bar: $50 \mu \mathrm{m}$. (C) Representative images of Masson's trichrome stain showing fibrosis in ilea of

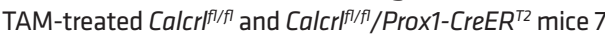
days after INDO challenge. $n=7-8$ animals in each group. Scale bar: $50 \mu \mathrm{m}$. (D) Relative expression of inflammatory markers in ilea of TAM-treated $\mathrm{Calcr}^{\mathrm{fl/fl}}$ and $\mathrm{Calcr}^{\mathrm{fl/fl} /}$ Prox1-CreER ${ }^{\text {T2 }}$ mice 7 days after INDO challenge. $n=7-8$ animals in each group. Quantitative data are represented as a box-and-whisker plot, with bounds from 25th to 75 th percentile, median line, and whiskers ranging from minimum to maximum values of fold change over Calcr $^{\mid f / / f l}$ controls from 3 independent studies. Gapdh was used as housekeeping control. Significance was determined by 2-tailed, type 2 Student's $t$ test, ${ }^{*} P<0.05$. (E) Quantification of small intestine length measured from proximal duodenum to distal ileum in Calcr $f^{f / f l}$ and Calcr $^{\text {fl/ff }} /$ Prox $1-C r e E R^{T 2}$ mice after TAM treatment, 1 day after INDO challenge, and 7 days after INDO challenge. $n$ = 5-10 mice per group. Quantitative data are represented as a box-and-whisker plot, with bounds from 25 th to 75th percentile, median line, and whiskers ranging from minimum to maximum values of gut length. Significance was determined by 2-tailed, type 2 Student's $t$ test, ${ }^{*} P<$ 0.05. (F) Histopathological score of inflammation based

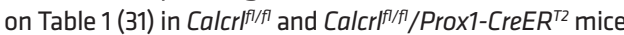
after TAM treatment, 1 day after INDO challenge, and 7 days after INDO challenge. $n=5-10$ mice per group. Quantitative data are represented as a box-and-whisker plot, with bounds from 25th to 75 th percentile, median line, and whiskers ranging from minimum to maximum inflammation scores. Significance was determined by 2-way ANOVA, ${ }^{*} P<0.05$.

absorption, leading to weight loss (32). Calcrtiffl/Prox1-CreER $R^{T 2}$ mice had significantly decreased body weight compared with control animals both 1 day and 7 days after INDO challenge, with chyle leakage and accumulation of lipid within the mesentery (Figure 5, A-C). Oil red O staining of Calcrtlffl/ Prox 1-CreER ${ }^{T 2}$ intestines revealed a striking accumulation of lipid molecules at the tips of the villous enterocytes, along with dramatically reduced lipid molecules toward the center of the villi or within the submucosal area compared with control animals (Figure 5D). These data illustrate that expression of CLR in lymphatics is essential for promoting lipid absorption within intestinal lacteals.

Numerous in vitro and in vivo studies have characterized a robust function for AM and CLR signaling in the regulation of endothelial permeability by reorganization of both the tight junction protein zonula occludens-1 (ZO-1) and adherens junction protein VE-Cadherin $(23,33,34)$. Therefore, we sought to determine whether disruption of lymphatic lacteal junctional proteins in Calcrtt/fl/Prox 1 $C r e E R^{T 2}$ animals could be associated with the failure of appropriate lipid absorption. Indeed, lacteals of Calcrltflf/Prox1-CreER ${ }^{T 2}$ animals displayed more continuous VE-Cadherin staining compared with controls, with no consistent change in cell shape or morphology (Figure 5E and Supplemental Figure $5 \mathrm{~A})$. These findings were further recapitulated in an in vitro system, whereby knockdown of $C A L C R L$ in human LECs resulted in more linearized localization of both VE-Cadherin and ZO-1 at cell-cell junctions, compared with the jagged cell-cell junctions exhibited in control siRNA-treated cells (Figure 

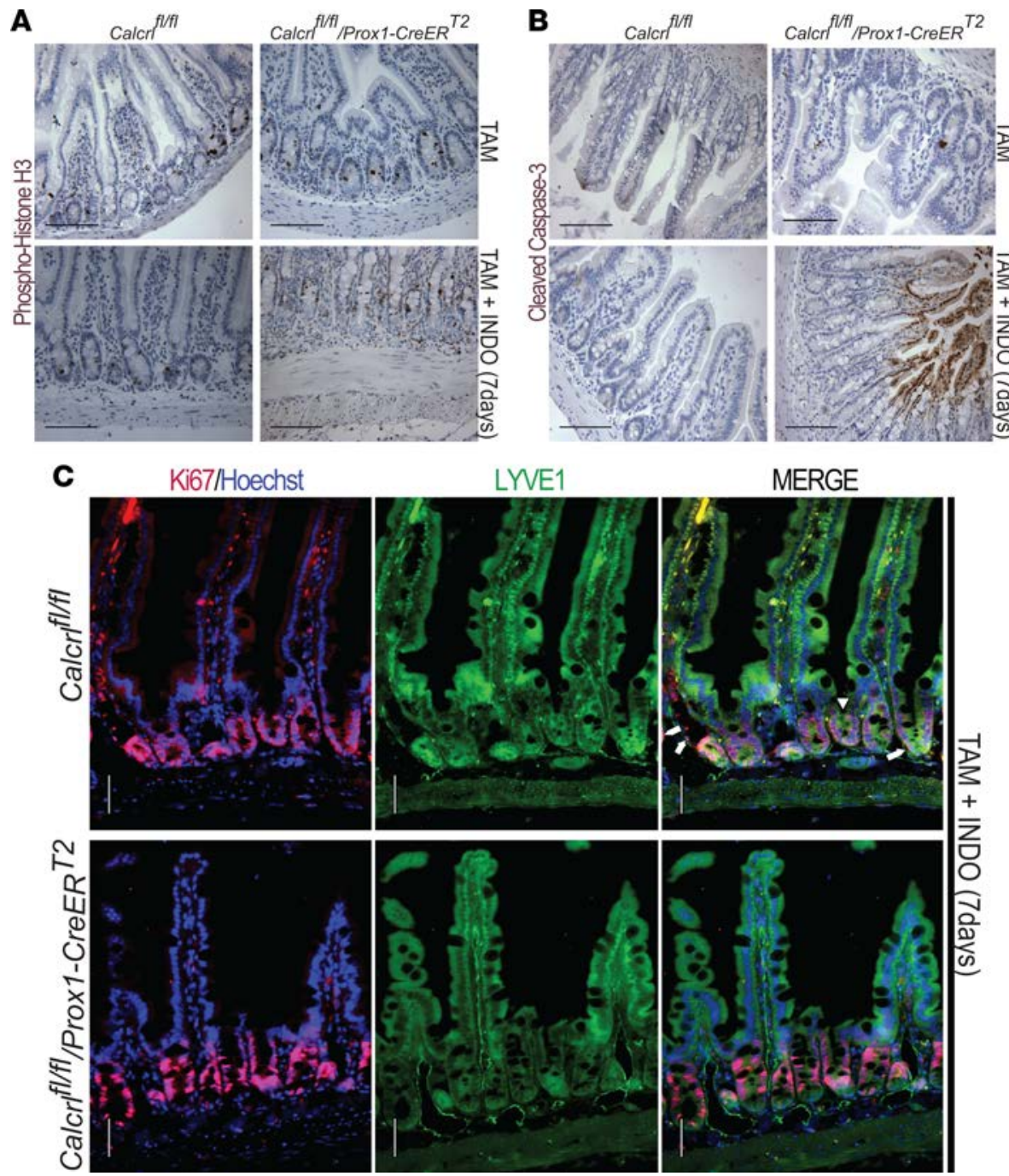

Figure 4. Increased proliferation in the crypts but diminished proliferation in lacteals lacking Calcrl. Representative images of proliferation marker phospho-histone H3stained (A) and apoptosis marker cleaved caspase-3-stained ilea (B) of tamoxifen-treated (TAM-treated) $\mathrm{Calcr}^{f / / f l}$ and $\left[\mathrm{Calcr} \mathrm{F}^{\mathrm{fl} / \mathrm{fl}} /\right.$ Prox1-CreER ${ }^{\text {T2 }}$ mice after TAM treatment and 7 days after indomethacin (INDO) challenge. (C) Representative images of proliferation marker Ki67- and lymphatic vessel endothelial hyaluronan receptor 1-stained (LYVE1-stained) ilea of TAM-treated Calcrl ${ }^{f / f l}$ and $\mathrm{Calcr}^{f / / f /} /$ Prox1-CreER ${ }^{T 2}$ mice 7 days after INDO challenge. Arrows point to the Ki67-positive area within the villi lacteal. Arrowhead indicates Ki67 staining in crypt. $n=7-8$ animals in each group. Scale bar: $50 \mu \mathrm{m}$.

$5 F)$. These results emphasize the influence of CLR signaling in intestinal lymphatic endothelial junctions and lipid uptake, which are independent of the ability of enterocytes to absorb lipids.

Notch signaling is downregulated in Calcrl-deficient LECs. Notch signaling has very recently been identified as an important mediator of intestinal inflammation and lacteal maintenance. Notch ligands DLL1 and DLL4 are expressed in intestinal crypts and are upregulated during inflammation (35), and continuous lymphatic-specific Dll4 expression induced by VEGFR2 and VEGFR3 is important in the regeneration and proper functioning of lacteals (6). Interestingly, in arterial endothelial cells, AM treatment robustly upregulates numerous Notch pathway components under conditions of hypoxia and regulates Notch pathway components in vascular progenitors during arterial differentiation $(36,37)$.

Therefore, consistent with these findings, we observed a pronounced increase in DLL4 in the crypts and whole intestine (Figure 6A, arrowheads, and Supplemental Figure 5B) - likely representative of the critical role Notch plays in intestinal stem cell proliferation and regeneration - while the Calcrt ${ }^{f l f t} /$ Prox 1-CreER ${ }^{T 2}$ lacteals had little to no DLL4 expression compared with control animals (Figure 6A, arrows and boxes). In addition, siRNA-mediated knockdown of CALCRL in human LECs showed downregulation of several Notch pathway components, along with downregulation of activated NOTCH1 and DLL4 by immunostaining (Figure 6, B-D). Conversely, treating human LECs with human AM peptide increased the expression of Notch components (Figure 6, E-G). Collectively, these data provide a critical cross-link between a robust lymphangiogenic signaling cue and its downstream effector pathway, both of which are essential for mediating intestinal lacteal integrity, function, and regeneration during intestinal inflammation. 
A

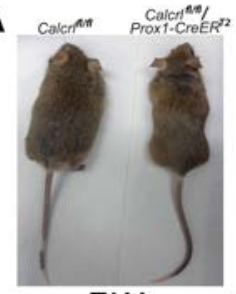

TAM
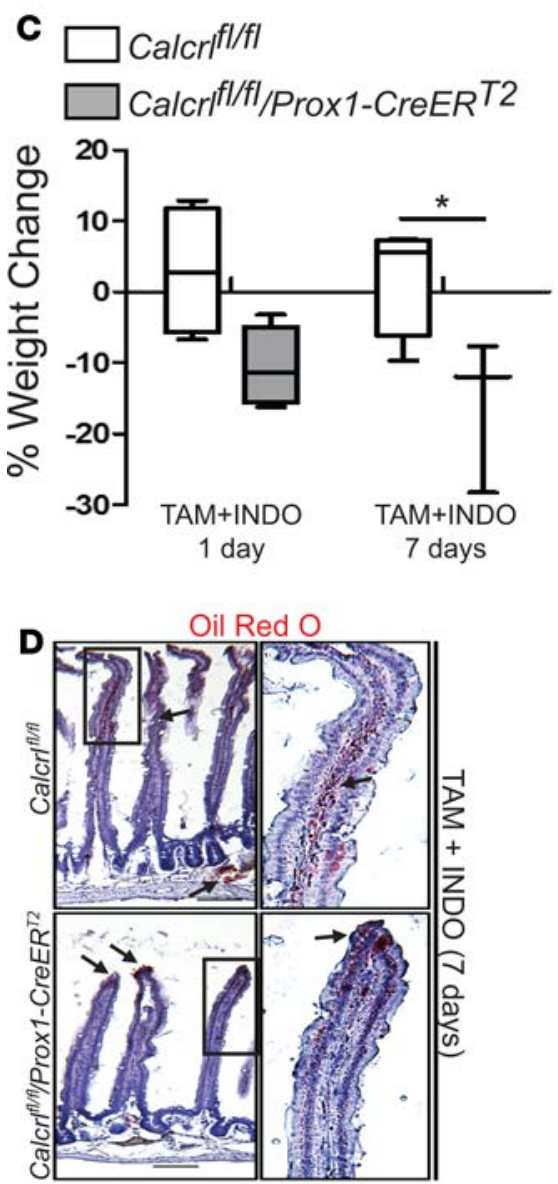
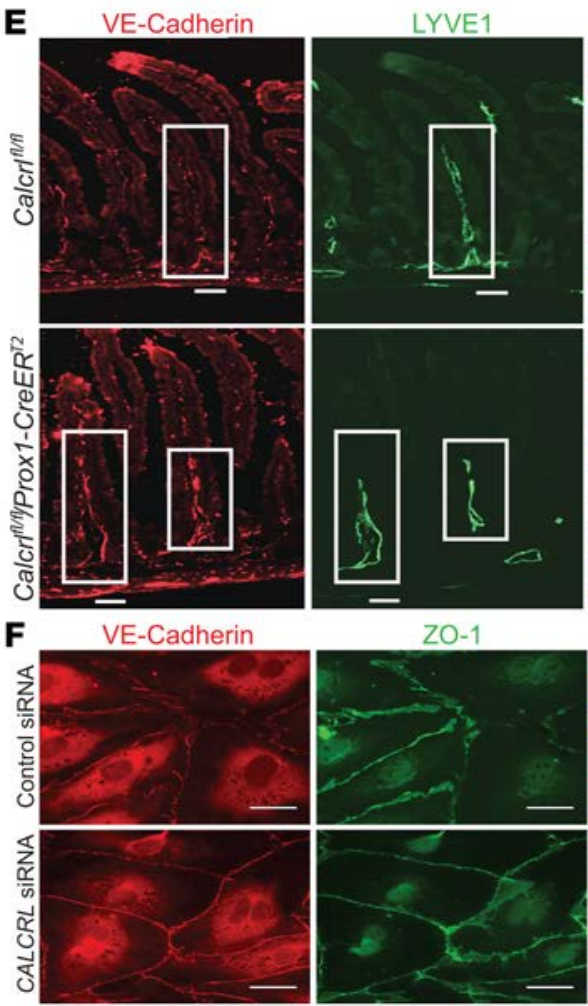

Hoechst/MERGE

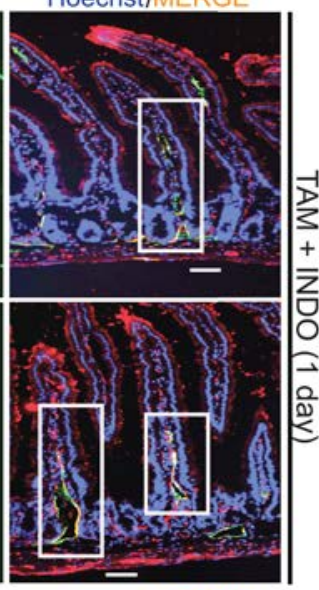

Hoechst/MERGE
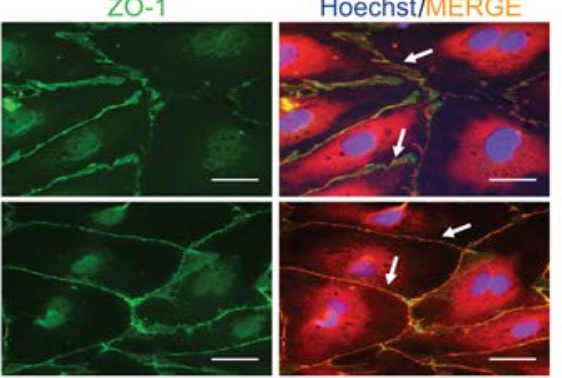

Figure 5. Impaired lipid uptake and junctional barriers in lymphatic Calcrl-deficient mouse intestines. (A) Images of tamoxifen-treated (TAM-treated) Cal-

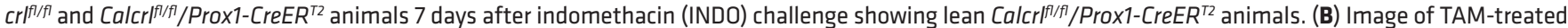
Calcr $^{\text {f/ff/ } / P r o x 1-C r e E R ~}{ }^{\text {T2 }}$ mouse abdomen and mesenteric region 7 days after INDO challenge. Arrow points to chyle leakage. (C) Measurement of weight loss in

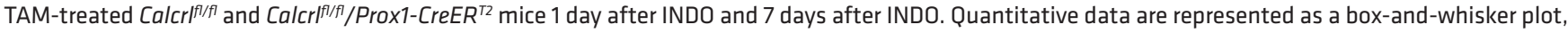
with bounds from 25th to 75th percentile, median line, and whiskers ranging from minimum to maximum percentage weight loss; $n=7-10$ animals per group per treatment. Significance was determined by 2-way ANOVA, ${ }^{*} P<0.05$. (D) Representative images of Oil red O-stained duodena of TAM-treated Calcr ${ }^{\text {fl/fl }}$ and Calcr $\left.\right|^{f / f / P} /$ Prox1-CreER ${ }^{T 2}$ mice 7 days after INDO challenge. $n=7-8$ animals in each group. Scale bar: $25 \mu \mathrm{m}$. Boxed regions are shown as zoomed insets to the right. Magnification: $\times 25$. Arrows highlight lipid droplets inside the villi or gathered at the top of the villi. (E) Representative images of VE-Cadherin- and

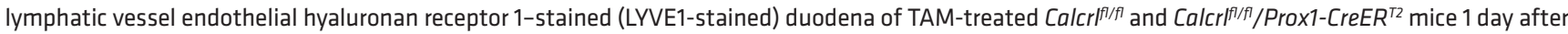
INDO challenge. $n=8-10$ animals in each group. Boxed regions highlight lacteals. Scale bar: $50 \mu \mathrm{m}$. (F) Representative VE-Cadherin- and zona occludens 1-stained (ZO-1-stained) images from 3 independent experiments in which human neonatal dermal lymphatic endothelial cells were treated with control siRNA or human calcitonin receptor-like receptor (CALCRL) siRNA. Scale bar: $100 \mu \mathrm{m}$. Arrows indicate junctions.

\section{Discussion}

There exists substantial controversy about whether lymphatic expansion during conditions of IBD occurs as an adaptive or aggravating response (3). The answer to this question is likely very complex and largely dependent on the stage of disease progression. For example, the expansion of lymphatics in discrete areas of the gut that are not histologically affected by inflammation suggests that lymphatic vessels probably 

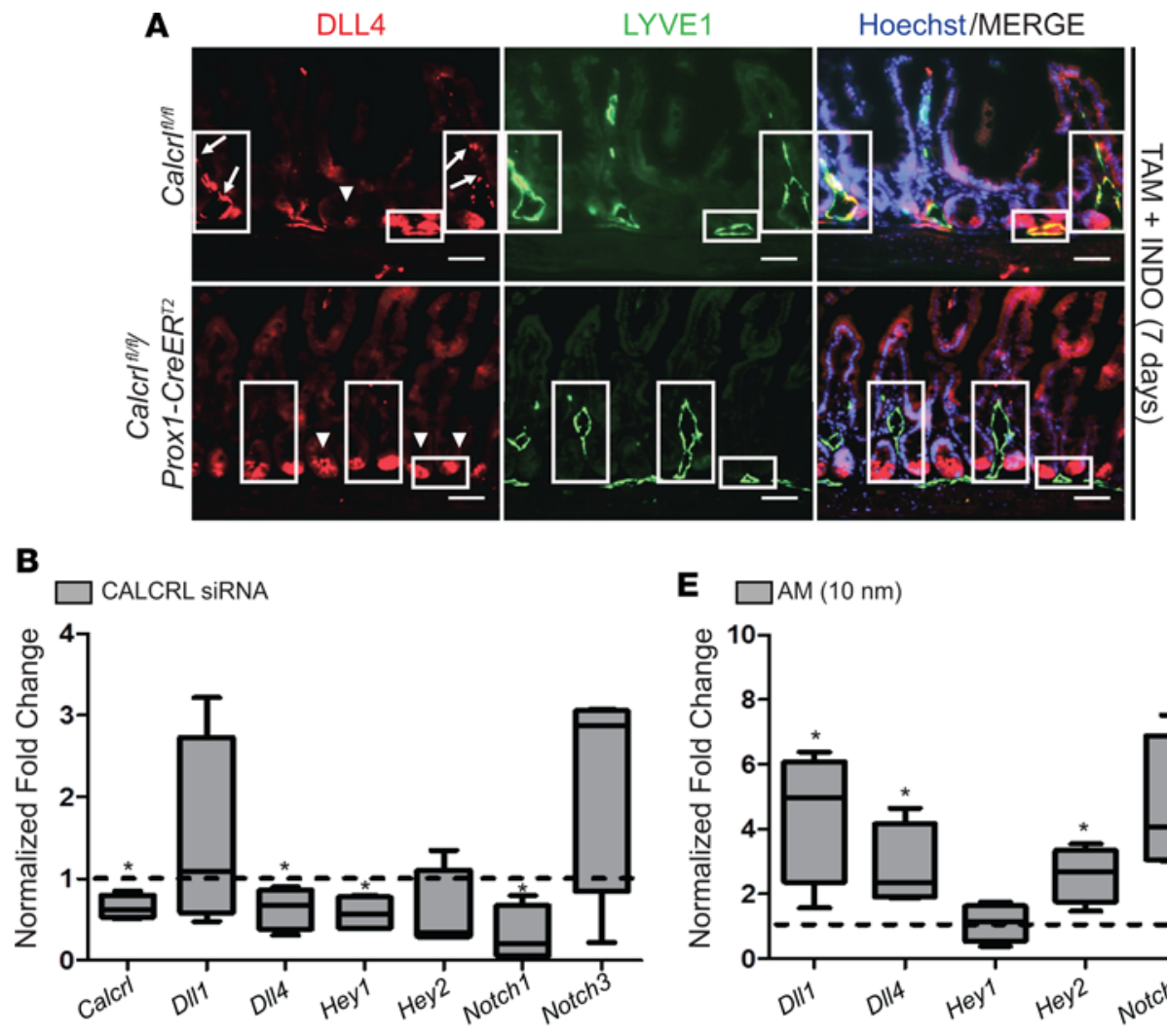

E $\square \mathrm{AM}(10 \mathrm{~nm})$
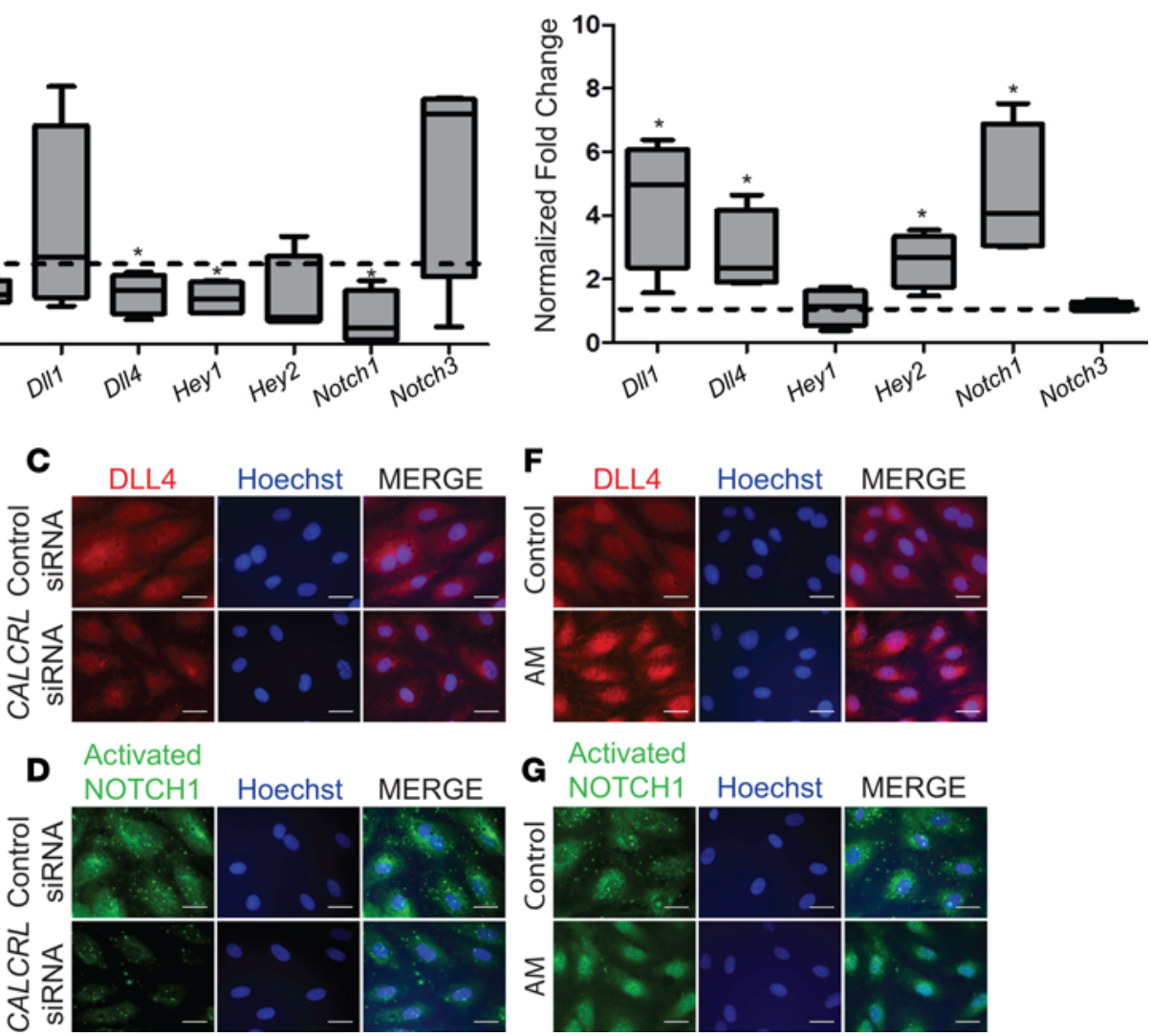

G $\begin{aligned} & \text { Activated } \\ & \mathrm{NOTCH} 1\end{aligned}$
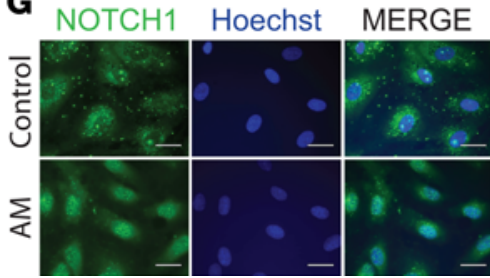

Figure 6. Notch signaling is downregulated upon loss of lymphatic Calcrl. (A) Representative images of delta-like canonical Notch ligand 4-stained (DLL4-stained) and lymphatic vessel endothelial hyaluronan receptor 1-stained (LYVE1-stained) ilea of tamoxifen-treated (TAM-treated) Calcr/f/fl and Calcr $F^{f / 1 / 7} /$ Prox1-CreER ${ }^{T 2}$ mice 7 days after indomethacin (INDO) challenge. $n=8-10$ animals in each group. Boxed regions highlight lacteals and submucosal lymphatic capillaries. Arrows point to DLL4 staining in lacteals. Arrowheads indicate DLL4 staining in crypts. Scale bar: $50 \mu \mathrm{m}$. (B and E) Relative expression of Notch pathway components in human neonatal dermal lymphatic endothelial cells after control siRNA or human calcitonin receptor-like receptor (CALCRL) siRNA treatment and vehicle (B) or $10 \mathrm{nM}$ adrenomedullin (AM) treatment (E). Quantitative data are represented as box-and-whisker plots, with bounds from 25th to 75th percentile, median line, and whiskers ranging from minimum to maximum normalized fold change over Calcr $f^{f / f l}$ from 4 independent studies. GAPDH, RN18S, and ACTB were used as housekeeping controls. Significance was determined by Student's $t$ test (tail $=2$, type $=2)$, ${ }^{*} P<0.05$. (C, D, F, and $\mathbf{G}$ ) DLL4-stained ( $\mathbf{C}$ and $\mathbf{F}$ ) and activated NOTCH1-stained ( $\mathbf{D}$ and $\mathbf{G}$ ) human neonatal dermal lymphatic endothelial cells after control siRNA or CALCRL siRNA treated with vehicle (C and $\mathbf{D})$ or $10 \mathrm{nM}$ AM treatment (F and $\mathbf{G})$. Images are representative of 3 independent knockdown experiments and treatment studies. Scale bar: $100 \mu \mathrm{m}$.

play an adaptive role during acute, subclinical phases of IBD (10). Moreover, the persistence of lymphangiectasia (but not blood vascular density) following intestinal recovery also suggests that lymphatics may play a protective role during the recovery phases of IBD by limiting inflammation (38). In contrast, rat and pig surgical models of lymphatic obstruction revealed marked regional enteritis and colitis with edema, 
Table 1. Histopathological scoring chart

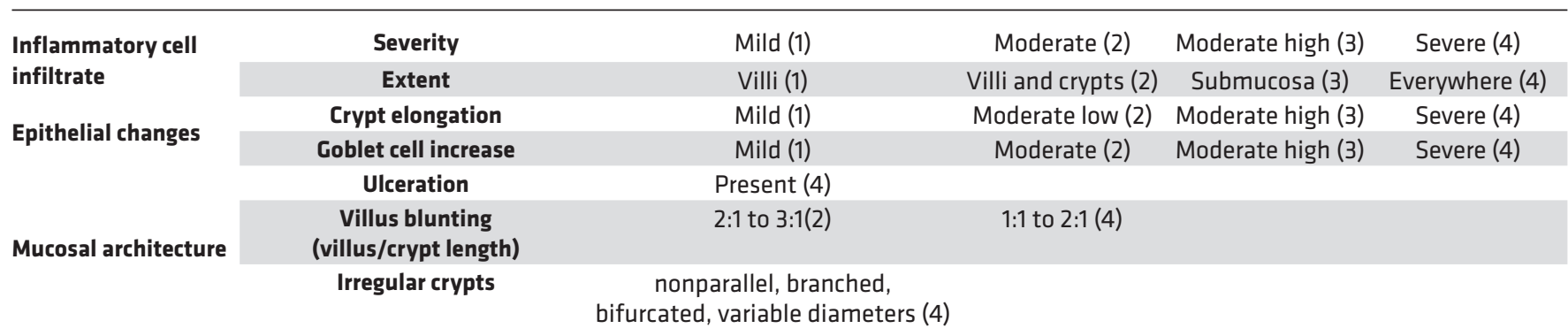

Adapted from the International Journal of Clinical and Experimental Pathology (31).

tissue remodeling, and increased inflammatory cytokines $(39,40)$, all of which clearly demonstrate that alterations in normal lymphatic function can be causative of IBD. Taken together, these studies support the concept that lymphangiectasia during early and recovery phases of IBD may be beneficial and that disturbances or stasis of lymphatic function during chronic IBD can exacerbate the condition and lead to an aggravated clinical presentation. Therefore, the genetic mouse model of lymphatic insufficiency presented herein provides us with an opportunity to further elucidate the functions of lymphatic vessels and AM signaling in mucosal injury and inflammation from several distinct perspectives.

Recently, using lymphatic-specific Dll4-null mice, it was shown that lymphatic DLL4 is essential for migration, lacteal tip cell survival, fat absorption, and regeneration of intestinal lacteals (6). Blocking DLL4 function using antibodies downregulates the Notch1-D114 axis and leads to dilation of collecting lymphatic vessels and impaired wound healing in adult mouse skin (41). In this study, we identify lymphatic Calcrl as an upstream regulatory factor that governs DLL4 expression to influence the resolution of inflammation after drug-induced acute intestinal injury. AM/CLR signaling has been shown to exert upstream regulation of the Notch pathway in the blood endothelium of zebrafish, and our current study substantiates the relevance of this pathway to the mammalian lymphatic vascular system in a disease model $(41,42)$. The lymphatic deletion of Calcrl caused dilated lacteals and protein-losing enteropathy that are characteristic of clinical intestinal lymphangiectasia. Moreover, lacteals in the intestines of these mice did not efficiently absorb lipids. Taken together, these studies identify a Calcrl-Notch-Dll4 cascade that is essential for maintained lymphatic function in intestines.

We next challenged these lymphatic insufficiency mice with INDO to induce a mucosal injury leading to acute inflammation in order to examine their ability to resolve the inflammation. NSAIDs, like INDO, are known to inhibit both COX-1 and -2 enzymes and induce direct cytotoxic effects on the epithelium (43). The dosage of INDO can be effortlessly regulated, does not effect survival, is clinically relevant, and provides a highly reproducible mouse model of intestinal injury (44). Although INDO challenge is an established method to induce severe enteropathy in animals and recapitulates some aspects of human CD (increased mucosal permeability and inflammation), it is an acute inflammation model, which allows for full recovery, unlike chronic IBD models. When challenged with an INDO-induced mucosal injury, these Calcrll/fl/Prox1-CreER $R^{T 2}$ mice exhibited persistent inflammation, which, unlike controls, did not fully resolve 7 days after injury, resulting in marked weight loss (Supplemental Figure 6). This is one of the first studies, to our knowledge, to evaluate the role of systemic lymphatic insufficiency on the progression and resolution of intestinal inflammation, and it underscores the critical importance of maintained lymphatic function for the proper response and resolution of acute intestinal inflammation. Additionally, it is important to note that most of the phenotype of persistent inflammation was observed in the ilea of TAM- and INDO-treated Calcrl/ft/Prox1-CreER ${ }^{T 2}$ mice, which is in tune with the high expression of preproAM mRNA in the ileum compared with other parts of the small intestine (45).

This study introduces a nonsurgical model of systemic lymphatic vascular insufficiency, currently lacking in the field (46). Future studies with prolonged injury models, such as ischemia/reperfusion, irradiation, or dextran sodium sulfate-induced colitis, will be very informative. Furthermore, this model of lymphatic insufficiency can be used to explore the nature of paracellular versus transcellular lipid transport pathways, central/nervous system innervation of lymphatics to control lipid uptake, and the crosstalk between intestinal LECs and enterocytes in lipid transport. Although there are some inbred strains of dogs (Wheaten Terrier, Lundehund, Basenji, and Yorkshire Terrier) that develop spontaneous 
intestinal lymphatic insufficiency, with secondary complications of intestinal inflammation and protein-losing enteropathy, to our knowledge the Calcr $f^{7 / f l} /$ Prox $1-C r e E R^{T 2}$ mouse model represents the first and only model in the field from which we can begin to address these broader questions (19).

The Notch ligand DLL4 was reduced in the lymphatic lacteals of Calcrll/fl/Prox1-CreER ${ }^{T 2}$ animals, which is the first evidence to our knowledge that AM/CLR signaling governs Notch signaling in LECs (Figure 6A). Consistent with inflammation, we found that DLL4 is upregulated in proliferative crypts of Calcrl/ff/Prox1CreER ${ }^{T 2}$ ileum (Figure 6A and Supplemental Figure 5B). However, at the same time, DLL4 was downregulated in the lacteals, implying that lymphatic CLR expression is not directly responsible for the crypt upregulation of DLL4 and proliferation. Additionally, increased epithelial proliferation did not affect the reduced proliferation of LECs. The dysfunctional lymphatics in Calcrt ${ }^{l / f t} /$ Prox $1-C r e E R^{T 2}$ mice exacerbated inflammation, even though the crypts followed the expected pattern of proliferation, emphasizing the importance of lymphatic vessels in disease progression and tissue repair. Therefore, our study also provides insights into the molecular crosstalk between LECs of dysfunctional lacteals and the villus enterocytes upon inflammation.

AM signaling through CLR and its roles on lymphatic vessel proliferation and junctional organization potentially provide a pharmacologically tractable target to resolve inflammatory diseases. Since more than $50 \%$ of all clinically available drugs target GPCRs (47), there is great interest in understanding pharmacological and biochemical properties of CLR and its associated RAMPs, with the ultimate goal of manipulating the GPCR/RAMP interface for treatment of human disease (48). The elucidation of specific roles for CLR in intestinal lymphatic function may ultimately form the basis of GPCR-targeted approaches for the therapeutic treatment of lymphatic vascular diseases or the amelioration of inflammatory digestive conditions through modulation of lymphatic function.

In summary, our study strongly supports the importance of lymphatic CLR in intestinal disease progression and restoration, which is mediated, in part, through its effect on Notch signaling. The induction of the intestinal lymphatic AM signaling pathway may therefore aid in enhanced intestinal inflammation resolution, thus presenting a potential target in the treatment of IBDs.

\section{Methods}

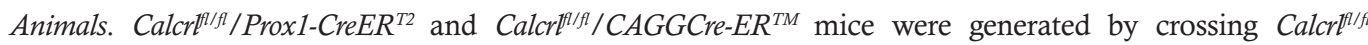
mice (22), which are on a background of N7-10 on C57BL/6, to either the Prox1-CreER ${ }^{T 2}$ line (24), a gift from Guillermo Oliver (Northwestern University, Chicago, USA) or CAGGCre-ER ${ }^{T M}$ [The Jackson Laboratory B6.Cg-Tg(CAG-cre/Esr1)5Amc/J]. WT/Prox1-CreER $R^{T 2} / R p l 22^{\text {tm1.1Psam }}$ (with WT Calcrl) and Calcrl ${ }^{\text {tl }}$ fl/Prox1-CreER $R^{T 2} / R$ pl22 tm1.1Psam mice were generated by crossing Calcrll/fl/Prox1-CreER ${ }^{T 2}$ mice with B6N.129$R p l 22^{\text {tml.1Psam }} / J$ mice, which are also known as RiboTag mice, from The Jackson Laboratory (stock 011029). Cre-mediated recombination was induced by administering TAM (Sigma-Aldrich T5648) dissolved in corn oil and ethanol to mice aged 4-6 months for 5 consecutive days at a dose of $5 \mathrm{mg} / 40 \mathrm{~g}$ intraperitoneally, after which organs were harvested. These mice were tested for floxed, Cre, and excised alleles by genotyping after TAM administration. Primers used for genotyping the WT Calcrl allele were 5'-GCGGAGCATATTCAATCACAAG-3' and 5'-GAAATGTGCTGTATGTTCAAGC-3', for the Calcrl floxed allele were 5'-GCGGAGCATATTCAATCACAAG-3' and 5'-GACGAGTTCTTCTGAGGGGA-3', for the Prox1-CreER ${ }^{T 2}$ allele were 5'-CGAGCTCTTTCTCTCTACAGTTCAACA-3' and 5'-GGCCAGTAACGTTAGGGAGAGG-3', for the excised allele were 5'-GCGGAGCATATTCAATCACAAG-3' and 5'-GAATAAGTTGAGCTGGG-

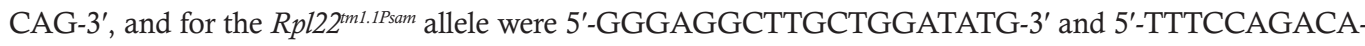
CAGGCTAAGTACAC-3'. Acute mucosal injury was induced by injecting INDO (Sigma-Aldrich I7378) dissolved in $5 \%$ sodium bicarbonate at $10 \mathrm{mg} / \mathrm{kg}$ body weight subcutaneously, following TAM injections, and then organs were harvested either 1 day after INDO (acute inflammation stage) or 7 days after INDO (recovery). A total of 61 male and female mice between 4 and 6 months of age were used in this study.

Cell culture. Human neonatal dermal LECs (HMVEC-dLyNeo-Der, Lonza CC-2812) were used within 8 passages and maintained on EGM-2MV bullet kit media (Lonza CC3202). For siRNA knockdown cells were seeded at $2 \times 10^{5}$ cells/well in 6-well plates and grown to $60 \%-80 \%$ confluency. Cells were transfected with either control siRNA (Santa Cruz Biotechnology sc-37007) or human CRLR siRNA (Santa Cruz Biotechnology sc-43705) at a concentration of $10 \mu \mathrm{M}$ for 48 hours or were treated with either $10 \mathrm{nM}$ human AM peptide (Bachem H-2932) in media or were treated with water (control) for 1 hour. For immunostaining, cells were plated in 2\% gelatin-coated (Sigma-Aldrich G1393) sterile glass coverslips and grown to an $80 \%$ confluent monolayer before siRNA KD or AM treatment. 
$R N A$ and quantitative RT-PCR. Lung and small intestinal tissue was collected in RNAlater (Ambion AM7021). RNA was extracted from whole tissue or cultured cells using TRIzol reagent (Ambion 15596026), followed by DNase (Promega M6101) treatment, and cDNA was prepared using iScript (Bio-Rad 170-8890). For harvesting cell-specific RNA from RiboTag mice, intestines from TAM-induced WT/Prox1-CreER ${ }^{T 2} /$ $R p l 22^{\text {tml.1Psam }}$ and Calcrll/fl $/$ Prox1-CreER $R^{T 2} / R p l 22^{\text {tm1.1Psam }}$ mice were harvested. The enterocyte fraction of ileum was separated from the nonenterocyte fraction as per Van Landeghem et al. (49). Briefly, tissue was placed in dissociation reagent 1 (PBS/30 mM EDTA/1.5 mMDTT) on ice for no longer than 15 minutes, followed by prewarmed dissociation reagent 2 (PBS/30 mM EDTA) with shaking to remove the submucosa and muscularis. The remaining sample was pelleted by centrifugation, and the cell pellets were used for harvesting HA-tagged RNA from Prox1-CreER ${ }^{T 2}$-expressing cells using immunoprecipitation using anti-HA.11 epitope tag antibody (BioLegend 901513) as per Sanz et al. (27). Quantitative RT-PCR was performed on a StepOnePlus (ABI) using Green-2-Go qPCR Mastermix (BioBasic B00098). Gene expression was assessed using human single-tube assays (Thermo Fisher Scientific/Applied Biosystems): RN18S (Hs99999901_s1), GAPDH (4310884E), ACTB (Hs99999903_m1), HEY1 (Hs01114113_m1), HEY2 (Hs00232622_m1), DLL1 (Hs00194509_m1), DLL4 (Hs00184092_m1), NOTCH1 (Hs01062014_m1), NOTCH3 (Hs01128541_m1), CALCRL (Hs00907738_m1), FLT1 (Hs01052961_m1), KDR (Hs00911700_m1), FLT4 (Hs01047677_m1) or mouse Gapdh (Mm99999915_g1), Calcrl(Mm00516986_m1), Dll4 (Mm0044619_m1), TNFa (Mm00443258_ m1), IL6 (Mm00446190_m1), and Mpo (Mm01298424_m1). The comparative $\Delta \Delta \mathrm{C}_{\mathrm{T}}$ method was used to analyze relative gene expression with ExpressionSuite Software (Thermo Fisher Scientific). Expression was normalized to housekeeping controls $G A P D H, A C T B$, and $R N 18 S$

Immunohistochemistry and immunofluorescence. Cells were fixed in 4\% PFA for 20 minutes; rinsed with 1× PBS; permeabilized with 1\% Triton X-100 in PBS for 15 minutes; blocked with $3 \%$ normal donkey serum (NDS) for 1 hour; and incubated with 1:100 goat anti-VE-Cadherin (sc-6358), mouse anti-ZO-1 (Invitrogen 339194), rabbit anti-DLL4 (Novus Biologicals NB600-892), or rabbit anti-activated Notch1 (Abcam ab8925) primary antibodies for 2 hours at room temperature. Secondary antibodies included donkey anti-rabbit Cy3 (Jackson ImmunoResearch 711-225-152), donkey anti-goat Cy3 (Jackson ImmunoResearch 7105-165-147), donkey anti-mouse Cy2 (Jackson ImmunoResearch 715-545-151), and Bisbenzimide H 33258 Hoechst (Sigma-Aldrich B1155) at 1:250. Small intestinal tissue was flushed with cold PBS, filleted open, and fixed in either $4 \%$ PFA or 10\% Zinc Formalin (Thermo Fisher Scientific PROTOCOL 23-313095) overnight. Whole mount adult intestines were stained using slightly modified previously published protocol (50). Briefly, 1-cm pieces of fixed ileum were permeabilized in PBST at $4^{\circ} \mathrm{C}$ overnight, washed, and blocked in 5\% NDS. Samples were then incubated in primary antibodies, rabbit anti-LYVE-1 (1:200, Fitzgerald 70R-LR005) and mouse antismooth muscle actin (1:200, Sigma-Aldrich A4700), for 4 days. Intestines were washed, blocked, and incubated for 48 hours in secondary antibodies, including donkey anti-mouse Alexa Fluor 488 (1:200, Jackson ImmunoResearch; catalog 715-545-151), donkey anti-rabbit Alexa 594 (1:200), and DAPI (1:500). Paraffin sections and cryosections were rehydrated, permeabilized using 1\% Triton X-100 in PBS for 20 minutes, and blocked in $5 \%$ NDS or $1 \%$ BSA when using tyramide signal amplification (TSA) reagent. For antibodies requiring antigen retrieval, slides were boiled for 20 minutes in $10 \mathrm{mM}$ citrate buffer using a microwave. Sections were incubated in primary antibodies, including anti-HA (BioLegend 901501), goat anti-DLL4 (R\&D Systems AF1389), goat anti-VE-Cadherin (sc-6358), rabbit anti-LYVE1 (1:300), mouse anti-smooth muscle actin (1:200), and rat anti-Ki67 (Dako M7248) at room temperature overnight. Sections were washed and incubated in secondary antibodies, including donkey anti-rabbit Cy3 (Jackson ImmunoResearch 711-225-152), Alexa Fluor 594 Tyramide (Thermo Fisher Scientific T20950), and donkey anti-goat HRP (Thermo Fisher Scientific A15999), along with TSA reagent and Hoechst33258 (Sigma-Aldrich B1155). The tissues and cells were mounted in Prolong gold (Life Technologies P36934). H\&E, Oil red O, rabbit anti-phospho-histone H3 (Cell Signaling Technology 9701), rabbit anti-cleaved caspase-3 (Cell Signaling Technology 9661), and Masson's trichrome staining were performed by the University of North Carolina Histology Research Core Facility.

Image acquisition. H\&E-, Masson's trichrome-, and Oil red $\mathrm{O}-$ stained slides were imaged using a Leitz Dialux 20 with QImaging Micropublisher 5.0 RTV color CCD camera. Fluorescence IHC images were acquired on a Nikon E800 fluorescence microscope with a Hammamatsu Orca CCD camera with Metamorph software (Molecular Devices Corp.). Whole mount fluorescent-stained ileum was imaged using a Leica M205FA fluorescent stereoscope with QImaging Micropublisher 5.0 RTV color CCD camera. Images were pseudocolored using Metamorph or ImageJ (NIH). Whole mount entire small intestines were imaged using a Nikon 5200 DSLR camera. 
Serum component analysis measurements. Blood was collected from submandibular bleed, allowed to clot at room temperature for 1 hour, and separated using Microtainer tubes (BD 50089794). Serum albumin level was analyzed by the Animal Clinical Chemistry and Gene Expression Lab at University of North Carolina. Serum alanine aminotransferase and alkaline phosphatase levels were analyzed using VetScan comprehensive diagnostic profile rotor (Abaxis 500-7123).

Statistics. A total of 61 mice were used in this study. All experiments were performed 3 or more times, and data are represented as mean with SEM. Significance was either by determined by 2-tailed, type 2 Student's $t$ test or 1-way ANOVA with Tukey's Multiple comparison test or 2-way ANOVA, with $P$ values of less than 0.05 considered significant.

Study approval. All animal procedures were approved by the University of North Carolina Chapel Hill's Institutional Animal Care and Use Committee, and all attempts were made to minimize pain and distress.

\section{Author contributions}

$\mathrm{RBD}$ and $\mathrm{KMC}$ conceived of the study and its design, drafted the manuscript, obtained funding, and provided study supervision; RBD and DOK acquired data, analyzed and interpreted data, and provided statistical analysis; RBD, KMC, and DOK provided critical revision of the manuscript for important intellectual content; ESB acquired data; and JBP provided technical support.

\section{Acknowledgments}

We thank Shengli Ding, Emily Moorefield, and Eric Blue for continuous consultation and technical assistance. We are very grateful to Kirk McNaughton, J. Ashley Ezzell, and Kara Clissold in the Histology Research Core Facility, Carolyn Suitt in the Center for Gastrointestinal Biology and Disease Histology Core, and Hyung-Suk Kim at the Animal Clinical Chemistry and Gene Expression Lab at University of North Carolina for specimen handling. We are grateful to all members of the Caron lab for helpful discussions. This study was supported by NIH grant DK099156 to KMC and American Heart Association grant 15POST25270006 to RBD.

Address correspondence to: Kathleen M. Caron, Department of Cell Biology and Physiology, 111 Mason Farm Road, 6312B Medical Biomolecular Research Building, CB\#7545, Chapel Hill, North Carolina 27599-7545, USA. Phone: 919.966.5215; E-mail: Kathleen_Caron@med.unc.edu.

1. Betterman KL, Harvey NL. The lymphatic vasculature: development and role in shaping immunity. Immunol Rev. 2016;271(1):276-292.

2. Choe $\mathrm{K}$, et al. Intravital imaging of intestinal lacteals unveils lipid drainage through contractility. J Clin Invest. 2015;125(11):4042-4052.

3. Alexander JS, Ganta VC, Jordan PA, Witte MH. Gastrointestinal lymphatics in health and disease. Pathophysiology. 2010;17(4):315-335.

4. Tso P, Balint JA. Formation and transport of chylomicrons by enterocytes to the lymphatics. Am J Physiol. 1986;250(6 Pt 1):G715-G726.

5. Jang JY, et al. Conditional ablation of LYVE-1+ cells unveils defensive roles of lymphatic vessels in intestine and lymph nodes. Blood. 2013;122(13):2151-2161.

6. Bernier-Latmani J, et al. DLL4 promotes continuous adult intestinal lacteal regeneration and dietary fat transport. J Clin Invest. 2015;125(12):4572-4586.

7. Nurmi H, Saharinen P, Zarkada G, Zheng W, Robciuc MR, Alitalo K. VEGF-C is required for intestinal lymphatic vessel maintenance and lipid absorption. EMBO Mol Med. 2015;7(11):1418-1425.

8. Miller MJ, McDole JR, Newberry RD. Microanatomy of the intestinal lymphatic system. Ann N Y Acad Sci. 2010;1207 Suppl 1:E21-E28.

9. Heatley RV, Bolton PM, Hughes LE, Owen EW. Mesenteric lymphatic obstruction in Crohn's disease. Digestion. 1980;20(5):307-313.

10. Pedica F, Ligorio C, Tonelli P, Bartolini S, Baccarini P. Lymphangiogenesis in Crohn's disease: an immunohistochemical study using monoclonal antibody D2-40. Virchows Arch. 2008;452(1):57-63.

11. Ganta VC, et al. Angiopoietin-2 in experimental colitis. Inflamm Bowel Dis. 2010;16(6):1029-1039.

12. von der Weid PY, Rehal S, Ferraz JG. Role of the lymphatic system in the pathogenesis of Crohn's disease. Curr Opin Gastroenterol. 2011;27(4):335-341.

13. Rahier JF, et al. Increased lymphatic vessel density and lymphangiogenesis in inflammatory bowel disease. Aliment Pharmacol Ther. 2011;34(5):533-543.

14. Rahier JF, et al. Decreased lymphatic vessel density is associated with postoperative endoscopic recurrence in Crohn's disease. Inflamm Bowel Dis. 2013;19(10):2084-2090.

15. Algaba A, et al. Relationship between levels of angiogenic and lymphangiogenic factors and the endoscopic, histological and clinical activity, and acute-phase reactants in patients with inflammatory bowel disease. J Crohns Colitis. 2013;7(11):e569-e579. 
16. D'Alessio S, et al. VEGF-C-dependent stimulation of lymphatic function ameliorates experimental inflammatory bowel disease. J Clin Invest. 2014;124(9):3863-3878.

17. Wang XL, Zhao J, Qin L, Cao JL. VEGFR-3 blocking deteriorates inflammation with impaired lymphatic function and different changes in lymphatic vessels in acute and chronic colitis. Am J Transl Res. 2016;8(2):827-841.

18. Wang XL, Zhao J, Qin L, Qiao M. Promoting inflammatory lymphangiogenesis by vascular endothelial growth factor-C (VEGF-C) aggravated intestinal inflammation in mice with experimental acute colitis. Braz J Med Biol Res. 2016;49(5):e4738.

19. Willard MD, et al. Intestinal crypt lesions associated with protein-losing enteropathy in the dog. J Vet Intern Med. 2000;14(3):298-307.

20. Caron KM, Smithies O. Extreme hydrops fetalis and cardiovascular abnormalities in mice lacking a functional Adrenomedullin gene. Proc Natl Acad Sci USA. 2001;98(2):615-619.

21. Dackor RT, Fritz-Six K, Dunworth WP, Gibbons CL, Smithies O, Caron KM. Hydrops fetalis, cardiovascular defects, and embryonic lethality in mice lacking the calcitonin receptor-like receptor gene. Mol Cell Biol. 2006;26(7):2511-2518.

22. Fritz-Six KL, Dunworth WP, Li M, Caron KM. Adrenomedullin signaling is necessary for murine lymphatic vascular development. J Clin Invest. 2008;118(1):40-50

23. Hoopes SL, Willcockson HH, Caron KM. Characteristics of multi-organ lymphangiectasia resulting from temporal deletion of calcitonin receptor-like receptor in adult mice. PLoS ONE. 2012;7(9):e45261

24. Srinivasan RS, et al. Lineage tracing demonstrates the venous origin of the mammalian lymphatic vasculature. Genes Dev. 2007;21(19):2422-2432.

25. Petrova TV, et al. Transcription factor PROX1 induces colon cancer progression by promoting the transition from benign to highly dysplastic phenotype. Cancer Cell. 2008;13(5):407-419.

26. Bjerknes M, Cheng H. Neurogenin 3 and the enteroendocrine cell lineage in the adult mouse small intestinal epithelium. Dev Biol. 2006;300(2):722-735

27. Sanz E, Yang L, Su T, Morris DR, McKnight GS, Amieux PS. Cell-type-specific isolation of ribosome-associated mRNA from complex tissues. Proc Natl Acad Sci USA. 2009;106(33):13939-13944.

28. Jin D, Otani K, Yamahara K, Ikeda T, Nagaya N, Kangawa K. Adrenomedullin reduces expression of adhesion molecules on lymphatic endothelial cells. Regul Pept. 2011;166(1-3):21-27.

29. Derelanko MJ, Long JF. Effect of corticosteroids on indomethacin-induced intestinal ulceration in the rat. Dig Dis Sci. 1980;25(11):823-829.

30. Yamada T, Deitch E, Specian RD, Perry MA, Sartor RB, Grisham MB. Mechanisms of acute and chronic intestinal inflammation induced by indomethacin. Inflammation. 1993;17(6):641-662.

31. Erben U, et al. A guide to histomorphological evaluation of intestinal inflammation in mouse models. Int J Clin Exp Pathol. 2014;7(8):4557-4576.

32. Holt Pr. Dietary Treatment of Protein Loss in Intestinal Lymphangiectasia. The Effect of Eliminating Dietary Long Chain Triglycerides on Albumin Metabolism in this Condition. Pediatrics. 1964;34:629-635.

33. Dunworth WP, Fritz-Six KL, Caron KM. Adrenomedullin stabilizes the lymphatic endothelial barrier in vitro and in vivo. Peptides. 2008;29(12):2243-2249.

34. Hocke AC, et al. Perturbation of endothelial junction proteins by Staphylococcus aureus alpha-toxin: inhibition of endothelial gap formation by adrenomedullin. Histochem Cell Biol. 2006;126(3):305-316.

35. Shimizu H, et al. Distinct expression patterns of Notch ligands, D111 and D114, in normal and inflamed mice intestine. PeerJ. 2014;2:e370.

36. Lanner F, et al. Hypoxia-induced arterial differentiation requires adrenomedullin and notch signaling. Stem Cells Dev. 2013;22(9):1360-1369.

37. Yurugi-Kobayashi T, et al. Adrenomedullin/cyclic AMP pathway induces Notch activation and differentiation of arterial endothelial cells from vascular progenitors. Arterioscler Thromb Vasc Biol. 2006;26(9):1977-1984.

38. Baluk P, et al. Pathogenesis of persistent lymphatic vessel hyperplasia in chronic airway inflammation. J Clin Invest. 2005;115(2):247-257.

39. Van Kruiningen HJ, Colombel JF. The forgotten role of lymphangitis in Crohn's disease. Gut. 2008;57(1):1-4.

40. Cavriani G, Domingos HV, Oliveira-Filho RM, Sudo-Hayashi LS, Vargaftig BB, de Lima WT. Lymphatic thoracic duct ligation modulates the serum levels of IL-1beta and IL-10 after intestinal ischemia/reperfusion in rats with the involvement of tumor necrosis factor alpha and nitric oxide. Shock. 2007;27(2):209-213.

41. Niessen K, et al. The Notch1-D114 signaling pathway regulates mouse postnatal lymphatic development. Blood. 2011;118(7):1989-1997.

42. Wilkinson RN, Koudijs MJ, Patient RK, Ingham PW, Schulte-Merker S, van Eeden FJ. Hedgehog signaling via a calcitonin receptor-like receptor can induce arterial differentiation independently of VEGF signaling in zebrafish. Blood. 2012;120(2):477-488.

43. Wallace JL. Prostaglandins, NSAIDs, and gastric mucosal protection: why doesn't the stomach digest itself? Physiol Rev. 2008;88(4):1547-1565

44. Fang WF, Broughton A, Jacobson ED. Indomethacin-induced intestinal inflammation. Am J Dig Dis. 1977;22(9):749-760.

45. Martínez-Herrero S, Martínez A. Adrenomedullin regulates intestinal physiology and pathophysiology. Domest Anim Endocrinol 2016;56 Suppl:S66-S83

46. Shin WS, Rockson SG. Animal models for the molecular and mechanistic study of lymphatic biology and disease. Ann $N Y$ Acad Sci. 2008;1131:50-74.

47. Drews J. Genomic sciences and the medicine of tomorrow. Nat Biotechnol. 1996;14(11):1516-1518.

48. Sexton PM, Poyner DR, Simms J, Christopoulos A, Hay DL. Modulating receptor function through RAMPs: can they represent drug targets in themselves? Drug Discov Today. 2009;14(7-8):413-419.

49. Van Landeghem L, et al. Activation of two distinct Sox9-EGFP-expressing intestinal stem cell populations during crypt regeneration after irradiation. Am J Physiol Gastrointest Liver Physiol. 2012;302(10):G1111-G1132.

50. Klein KR, et al. Decoy receptor CXCR7 modulates adrenomedullin-mediated cardiac and lymphatic vascular development. Dev Cell. 2014;30(5):528-540. 